\title{
Acute subdural hematoma and subarachnoid hemorrhage associated with cerebral venous sinus thrombosis in apparently healthy individual
}

\author{
Reddy V. Umamaheswara', M. Venkatesh'1, Sai Krishna1, Amit Agrawal² \\ ${ }^{1}$ Department of Radiology, Narayana Medical College Hospital, Chinthareddypalem, Nellore, AP, India \\ ${ }^{2}$ Department of Neurosurgery, Narayana Medical College Hospital, Chinthareddypalem, \\ Nellore, AP, India
}

\begin{abstract}
Cortical venous thrombosis is one of the less common types of strokes. Diagnosis is usually delayed due to a wide spectrum of clinical manifestations. Subdural hemorrhage with subarachnoid hemorrhage secondary to cerebral venous sinus thrombosis is very rare. We report a case of 65 -year-old male with cerebral venous sinus thrombosis who presented to us with an acute subdural hematoma and subarachnoid hemorrhage associated with venous infarct. Management of such patients is complicated due to contraindication for the use of anticoagulation.
\end{abstract}

Keywords: cerebral venous sinuses, venous infarct, thrombosis, subdural hemorrhage

\section{INTRODUCTION}

Cerebral venous thrombosis involves thrombosis of the dural sinus and/or cortical veins (1). Patients usually present with a severe headache or focal neurological deficit but less frequently may present as acute neurological deterioration which can be fatal (1-5). We report a case of 65-year-old male who presented with an acute onset headache and acute confusional state. He was diagnosed to have transverse and sigmoid sinus thrombosis with left acute fronto-temporo-parietal subdural hematoma with venous infarct.

\section{CASE REPORT}

A 65 year old male patient not a known diabetic, hypertensive, smoker or alcoholic presented with sudden onset severe headache with nopostural variation with increasingly ascending in nature. Fol- lowed by acute confusional state was brought to our emergency room. Magnetic resonance imaging (MRI) scan was performed in view of clinical suspicion of cerebral venous thrombosis. MRI was performed on GE HDxt 1.5 T MRI machine with Spin echo sequences, venogram and Susceptibility weighted imaging, which revealed left temporal lobe hemorrhagic venous infarct with left transverse and sigmoid sinus thrombosis and thin left fronto-temporo-parietal convexity subdural hemorrhage and minimal sulcal subarachnoid hemorrhage, there was no significant mass effect or midline shift (Fig. 1). Patient was managed conservatively with LMWH and mannitol, his headache was relieved, patient was discharged on oral anticoagulation. One month later he came back to our hospital with similar symptoms and MRI was performed which revealed thrombosis of superior sagittal sinus, left transverse, sigmoid sinus 
and left internal jugular vein with right frontoparietal lobe venous infarct with hemorrhage and subdural hemorrhage along left fronto-temporo-parietal convexity was resolved (Fig. 2). Patient was managed conservatively with LMWH every 12 hours and followed by oral anticoagulation.

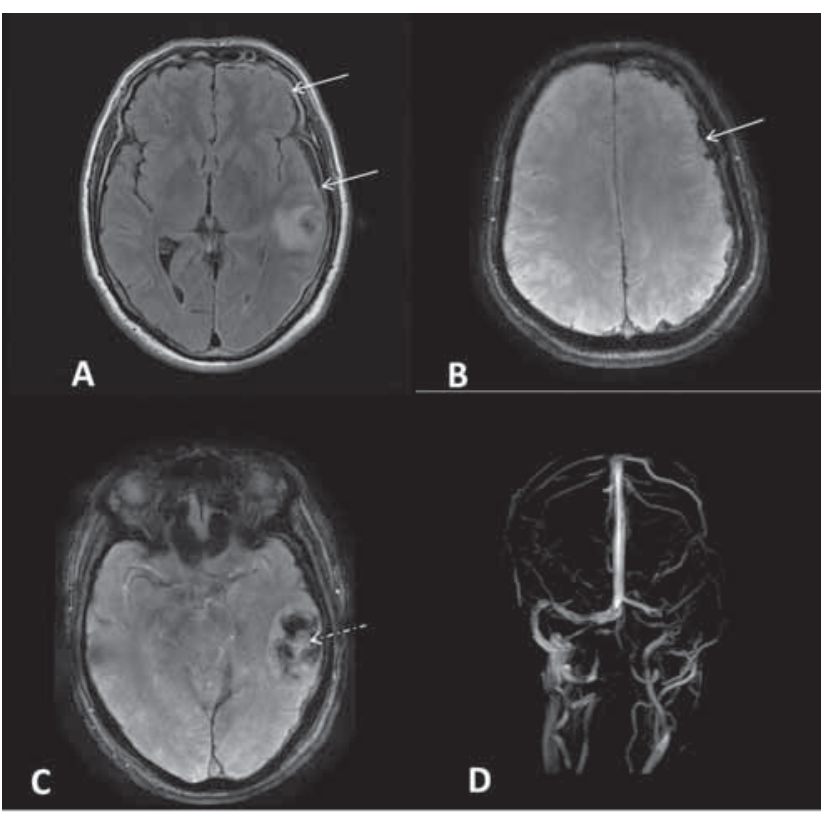

FIGURE 1. Initial MRI axial FLAIR (A) and Axial SWI (B) showing hyperintense subdural hemorrhage with blooming (arrows) along left frontotemporal convexities with hemorrhage on axial SWI (C) showing hemorrhage in left temporal lobe. MRV (D) shows thrombosis of left transverse, sigmoid sinuses and internal jugular vein.

\section{DISCUSSION}

Cerebral venous thrombosis accounts for 0.5$1 \%$ of stroke patients. More commonly seen in young patients than in elderly. Cerebral venous occlusion can occur due to intraluminal thrombus or extrinsic compression. Various causes of thrombosis like dehydration, pregnancy, usage of contraceptive pills, trauma, inflammatory diseases, infections, diabetes mellitus, nephrotic syndrome and neoplasms are implicated (3). Clinical features of venous sinus thrombosis are variable and is always challenging to the clinician where active search of venous sinuses and cortical veins is of utmost importance.

Our patient presented with severe headache and altered sensorium where imaging revealed thrombosis of venous sinuses complicated by subdural hemorrhage. First report of subdural hemorrhage complicating cerebral venous thrombosis was published by Bucy et al (2).

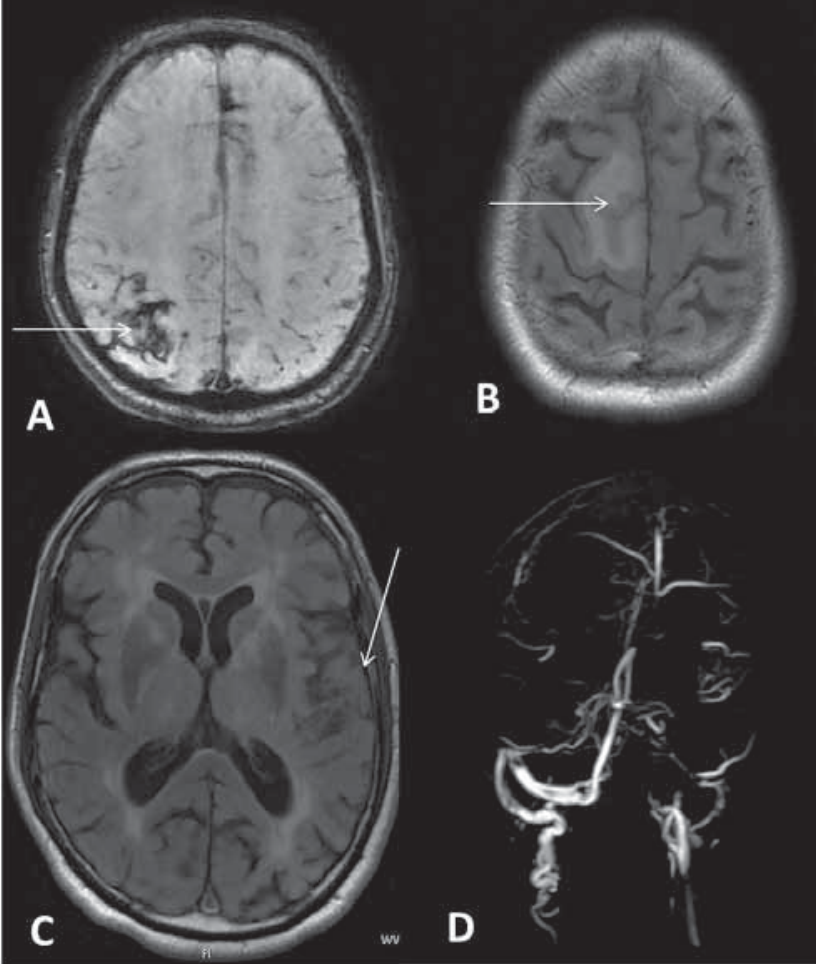

FIGURE 2. One month later MRI was done. Axial SWI (A) and FLAIR $(B, C)$ showing venous infarct with hemorrhage in right frontoparietal lobes with resolved subdural hemorrhage along left frontotemporal convexities. MRV (D) shows thrombosis of left transverse, sigmoid sinuses. left internal jugular vein and superior sagittal sinus

Previously acute subdural hemorrhage due to sinus thrombosis was commonly seen in neonates and infants. Blockage of venous drainage leads to high back pressure which leads to rupture of bridging veins resulting in subdural hemorrhage, subarachnoid hemorrhage and intraparenchymal hemorrhage $(4,5)$.

Imaging plays a major role in diagnosis and management of complicated cases of venous sinus thrombosis. MRI with MR Venogram is more definitive imaging modality for diagnosing amd detecting complications due to venous sinus thrombosis. CT venogram is an excellent imaging modality for detection of thrombi within the dural venous sinuses and due to radiation exposure it is reserved for the cases where MRI with MRV is not possible or where MRI is contraindicated (1). Management of patients with venous sinus thrombosis complicated by subdural hemorrhage is very difficult and should be cautious. In the present day, low molecular weight heparin is usually given in acute stages followed by oral anticoagulation for few months. 


\section{CONCLUSION}

Subdural hematoma secondary to cerebral venous sinus thrombosis is very rare, and high suspicion should be kept in high-risk cases and identifying this condition is extremely important as treatment options differ. Cerebral venous thrombosis presenting with spontaneous $\mathrm{SDH}$ is a rare entity. In our case lack of history of trauma and associated severe headache prompted us to perform an MR venogram which helped us in making the diagnosis.

Conflict of interest: none declared Financial support: none declared

\section{REFERENCES}

1. Krasnokutsky M.V. Cerebral venous thrombosis: a potential mimic of primary traumatic brain injury in infants. American Journal of Roentgenology 2011; 197:W503-W507.

2. Akins P.T., Axelrod Y.K., Ji C. et al. Cerebral venous sinus thrombosis complicated by subdural hematomas: Case series and literature review. Surgical neurology international 2013; 4.

3. Bansal H., Chaudhary A., Mahajan A., Paul B. Acute subdural hematoma secondary to cerebral venous sinus thrombosis: Case report and review of literature. Asian journal of neurosurgery 2016; 11:177.

4. Khatib K., Baviskar A. Treatment of cerebral venous sinus thrombosis with subdural hematoma and subarachnoid hemorrhage. Journal of Emergencies, Trauma and Shock 2016; 9.

5. Takahashi S., Shinoda J., Hayashi T. Cerebral venous sinus thrombosis in an adult patient presenting as headache and acute subdural hematoma. Journal of Stroke and Cerebrovascular Diseases 2012; 21:338-340. 